\title{
Mean Platelet Volume and Unresectable Hepatocellular Carcinoma
}

\author{
Satvinder Singh Bakshi \\ Department of ENT and Head and Neck Surgery, All India Institute of Medical Sciences \\ Mangalagiri, Guntur, India
}

\section{Dear Editor,}

I read with great interest the article "Association of Platelet Count and Mean Platelet Volume with Overall Survival in Patients with Cirrhosis and Unresectable Hepatocellular Carcinoma" by Scheiner et al. [1]. The study is interesting; however, I would like to point out certain issues regarding the mean platelet volume.

Firstly, many factors influence the mean platelet volume, such as diabetes, obesity, hypertension metabolic syndrome, and even the use of certain antihypertensives [2]. The presence of these conditions will definitely confound the results, and they should have been excluded from the study population.

Secondly, although it is relatively easy to obtain the mean platelet volume, the underlying principle of its measurement influences the results; therefore, the results will vary from the type of machine used [3]. In addition, the values also change depending on the duration between venipuncture and analysis and the type of anticoagulant used [3]. Thirdly, there are age and sex variations in the mean platelet volume, which could also be taken into account [4]. With so many confounding factors, it is difficult to make any conclusion, and a prospective study with a control population using the standard technique may give us a clear idea of the prognostic value of the mean platelet volume in hepatocellular carcinoma.

\section{Disclosure Statement}

The author has no conflicts of interest to disclose.

\section{Funding Sources}

No funding was obtained. 


\section{References}

1 Scheiner B, Kirstein M, Popp S, Hucke F, Bota S, Rohr-Udilova N, et al. Association of Platelet Count and Mean Platelet Volume with Overall Survival in Patients with Cirrhosis and Unresectable Hepatocellular Carcinoma. Liver Cancer. 2019 May;8(3):203-17.

2 Vizioli L, Muscari S, Muscari A. The relationship of mean platelet volume with the risk and prognosis of cardiovascular diseases. Int J Clin Pract. 2009 Oct;63(10):1509-15.

3 Beyan C, Beyan E. Were the measurements standardized sufficiently in published studies about mean platelet volume? Blood Coagul Fibrinolysis. 2017 Apr;28(3):234-6.

4 Ali U, Gibbs R, Knight G, Tsitsikas D. Sex-divided reference intervals for mean platelet volume, platelet large cell ratio and plateletcrit using the Sysmex XN-10 automated haematology analyzer in a UK population. Hematol Transfus Cell Ther. 2019 Apr - Jun;41(2):153-7. 